\title{
Effects of rule pretraining on rule effects in an attribute identification task*
}

\author{
LINDA J. INGISON $\dagger$ \\ University of Colorado, Boulder, Colorado 80302
}

\begin{abstract}
Rule learning (RL) training or familiarization as a means of reducing attribute identification (AI) rule effects was examined. Independent groups of college Ss were trained on 0, 2, 4, or $8 \mathrm{RL}$ problems based on the conjunctive $(\mathrm{Cj})$, disjunctive $(\mathrm{Dj})$, conditional $(\mathrm{Cd})$, or biconditional $(\mathrm{Bd})$ rules prior to transfer to an AI task based on the same rule. Contrary to predictions, level of pretraining failed to interact with rule difficulty in $\mathrm{AI}[\mathrm{F}(9,32)=1.90, \mathrm{p}<.25]$, while the main effect of rule obtained significance $[F(3,32)=10.12, p<.01]$. Implications of these findings for research conceived within a model postulating independent processes for the $\mathrm{RL}$ and $\mathrm{AI}$ components of concept learning tasks were discussed.
\end{abstract}

Haygood and Bourne (1965) presented data in general support of a model which postulates independent processes for the $\mathrm{AI}$ and $\mathrm{RL}$ components of concept learning. Since then, data have accumulated rapidly in general support of such independent $\mathrm{RL}$ and $\mathrm{AI}$ components. A number of studies have shown that certain variables (e.g., effects of positive and negative information; amount of irrelevant information) produce uniquely different effects when studied in RL as opposed to AI (Bourne \& Guy, 1968b; Haygood \& Stevenson, 1967; Bower \& King, 1967; Kepros \& Bourne, 1966).

In contrast, studies assessing rule 'difficulty have revealed essentially identical functions in both RL and AI. Previous experiments (Bourne, 1967, 1970; Haygood \& Bourne, 1965; Bourne \& Guy, 1968a; Conant \& Trabssso, 1964; Di Vesta \& Walls, 1969) have shown that, for a naive $S$, the specific rule determines the relative difficulty of both $\mathrm{RL}$ and $\mathrm{AI}$ problems. Furthermore, the order of difficulty of at least the rules utilized in the present study is the same for both tasks. Such findings appear to contradict Haygood and Bourne's (1965) analysis, since by this rule, effects would logically be expected only in RL.

One possible determinant of rule effects in $\mathrm{AI}$ is the difficulty that Ss may experience in fully understanding the given rule from instructions. Typical AI instructions are primarily concerned with providing information about the rule. Particularly when highly unfamiliar rules are used, it may be quite difficult for $S$ to grasp the given information merely through instructions.

*This research was undertaken within the Institute for the Study of Intellectual Behavior and is Publication No.45 of the institute. The work was supported by Research Grant No. MH 14314 for the National Institute of Mental Health. The author wishes to thank Judi Taddonio for her assistance in executing the study as well as Jed Docherty and Joel Level for their helpful comments on an earlier version of this paper. This paper is sponsored by Peter $\mathrm{J}$. Kepros, who takes full editorial responsibility for its contents.

+Requests for reprints should be sent to Linda J. Ingison, Wisconsin Research and Development Center for Cognitive Learning, University of Wisconsin, 1025 West Johnson Street. Madison, Wisconsin 53706.
Therefore, during the AI task, some residual rule learning may be taking place.

An alternative to simple rule instructions is rule familiarization or practice. Since practice on several successive RL problems has been shown to eliminate rule differences (Bourne \& Guy, 1968a; Bourne, 1970), high levels of RL pretraining may eliminate most or all of the rule learning involved in AI. If residual rule learning is the major factor producing rule effects in AI, Ss highly trained on a particular rule should reveal few, if any, rule differences on an AI transfer task. The present study was designed to assess this hypothesis.

\section{METHOD}

\section{Subjects and Design}

Ss were 48 volunteers, serving for credit in introductory psychology classes at the University of Colorado. Each S was assigned in order of appearance at the laboratory to one of 16 conditions by means of randomized sequences designed to place one $S$ in each cell before placing a second $S$ in any cell. Three Ss were assigned to each condition, without regard to sex. The design was a 4 by 4 factorial, involving four rules $(\mathrm{Cj}, \mathrm{Dj}, \mathrm{Cd}$, and $\mathrm{Bd})$ and four levels of pretraining $(0,2,4$, and $8 \mathrm{RL}$ problems).

\section{Stimuli and Problems}

The stimuli chosen were 27 geometric patterns, resulting from the enumeration of 3 trileveled dimensions. The dimensions used were size (large, medium, and small), shape (triangle, square, and hexagon), and color (red, yellow, or blue).

A total of nine problems ( $8 \mathrm{RL}$ and $1 \mathrm{AI})$ were called for by the design. To avoid redundancy in use of relevant attributes, selection was restricted to the use of each dimension six times, and each attribute on any dimension twice. Two problems involving the same pair of relevant dimensions were not presented in succession, and the final problems prior to transfer involved the same relevant attributes for all pretraining conditions.

The amount of logical information presented was the same per block of trials regardless of the rule involved. For RL problems, each set of four trials was a random arrangement of stimuli drawn from the four truth table classes (TT, TF, FT, and FF). For AI, the minimal number of stimuli logically needed to solve was identified for each rule. In addition to this minimal unit of information, at least one instance from each truth table category, as well as an equal number of positive and negative 
Table 1

Error Means on the AI Transfer Task for the Nonsignificant Interaction of Pretraining and Rule

\begin{tabular}{crrrrr}
\hline & \multicolumn{5}{c}{ Pretraining: RL Problems } \\
\cline { 2 - 5 } Rule & \multicolumn{1}{c}{0} & \multicolumn{1}{c}{2} & \multicolumn{1}{c}{4} & \multicolumn{1}{c}{8} & \\
\hline $\mathrm{Cj}$ & 2.00 & 1.00 & .33 & 0 & .58 \\
$\mathrm{Dj}$ & 8.67 & 3.67 & 3.00 & 5.00 & 5.08 \\
$\mathrm{Cd}$ & 8.00 & 17.00 & 22.33 & 4.00 & 12.83 \\
$\mathrm{Bd}$ & 17.00 & 15.67 & 13.33 & 7.33 & 13.33 \\
& 8.67 & 9.33 & 9.75 & 4.08 & \\
\hline
\end{tabular}

instances were presented. The resulting informational units were eight trials long.

\section{Procedure}

Typed instructions and appropriate example cards for the RL task were presented to $S$. Stimuli were presented on slides and rear projected onto a screen with a Kodak Carousel projector. A modified reception paradigm was employed in which study trials (answers given) were alternated with test trials (in which $\mathrm{S}$ was required to classify each pattern). Study and test sequences alike consisted of one instance from each truth table class. The Ss responded on test trials be pressing one of two buttons labeled "yes" and "no" on a box in front of the screen. After each response, a light appeared above the correct button (regardless of S's response) as feedback for $\mathrm{S}$. Slide and feedback were presented for $2 \mathrm{sec}$, after which feedback alone was present for $2 \mathrm{sec}$. On study trials, the light indicating the correct response appeared with the slide, and slide plus feedback were present for a total of $5 \mathrm{sec}$. Up to 72 test trials were given on each RL problem to reach a criterion of 16 correct test trials in a row. Those Ss not reaching this criterion were given the solution by $\mathrm{E}$, and required to demonstrate this solution by reaching the criterion of 16 correct. At this time, $S$ was taken to the next problem. Thus, no Ss were eliminated from the analysis. A total of $7 \mathrm{Ss}$ failed to solve the first RL problem. No S failed to solve any subsequent RL problem.

At completion of all RL problems, $\mathrm{S}$ was instructed as to the nature of the AI task. For AI, eight study trials (one complete informational unit) alternated with four test trials (a TT, TF, FT, and FF) up to a maximum of 72 test trials. A total of two Ss failed to solve the AI task. No S failed to solve both an RL and the AI problem.

\section{RESULTS}

In order to determine that a high level of rule familiarization had eliminated rule differences prior to transfer to the AI task, an analysis of variance on rule was computed on errors for the eighth RL problem. As predicted, the main effect of rule did not reach significance, $F(3,8)=1.97, p<.25$.

Transfer performance was analyzed by means of a 4 (pretraining conditions) by 4 (rules) analysis of variance on errors to criterion. Contrary to the prediction that increasing amounts of RL training would significantly reduce or eliminate rule effects in $\mathrm{AI}$, the interaction of pretraining and rule did not reach significance, $F(9,32)=$ $1.52, \mathrm{p}<.25$. Table 1 presents the means associated with this effect.

The main effect of rule also attained significance, $F(3,32)=10.12, p<.01$. Error means for each rule were $.58(\mathrm{Cj}), 5.08(\mathrm{Dj}), 12.83(\mathrm{Cd})$, and $13.33(\mathrm{Bd})$, indicating, in agreement with previous studies (Haygood
\& Bourne, 1965; Bourne \& Guy, 1968a), the difficulty of the $\mathrm{Cd}$ and $\mathrm{Bd}$ rules as compared to the $\mathrm{Cj}$ and $\mathrm{Dj}$. No other effects reached significance. In sum, the results show that for the pretraining levels employed in the present study, rule effects in $\mathrm{AI}$ are not eliminated through RL training.

\section{DISCUSSION}

On the basis of Haygood and Bourne's (1965) analysis of concept learning in terms of independent $\mathrm{RL}$ and $\mathrm{AI}$ components, it was predicted that pretraining on several RL problems would reduce or eliminate rule effects in a subsequent AI task. As evidenced by the nonsignificant interaction of rule and pretraining, however, RL training has little or no effect in reducing rule differences in $\mathrm{AI}$.

In light of these results, the nature of the critical factor(s) involved in AI rule effects is still in question. One possibility is that strategies specific to each underlying rule must be developed with practice on AI tasks involving the same rule. Optimal selection strategies have been indentified which develop with practice on successive problems involving the same rule (Laughlin, 1968; Laughlin \& Jordan, 1967), although such strategies do not appear powerful enough to eliminate rule effects (Taplin \& Jeeves, 1972). Such findings, however, do not rule out the likelihood that optimal reception strategies, when fully developed, may reduce rule effects.

Additional possibilities include more varied or more extensive training. The development of a varied repertoire of skills may be the key to effecting a reduction in rule differences in AI. It is unclear at this time what skills would be included in such a repertoire, al though some obvious candidates are overtraining on the same or a variety of rules (in RL, AI, or both), and specific truth table training.

Should the possession of rule-specific AI strategies or a repertoire of skills prove ineffective in reducing rule effects in AI, the implications are twofold. Models proposing independent processes for RL and AI may have to be revised to account for an interactive component. Further, research based on a two-component model may have to be interpreted with some caution, since at least for some variables it may be premature to conclude that reuslts are attributable to simple $\mathrm{RL}$ and $\mathrm{AI}$ processes.

\section{REFERENCES}

Bourne, L. E., Jr. Learning and utilization of conceptual rules. In B. Kleinmuntz (Ed.), Memory and the structure of concepts. New York: Wiley, 1967.

Bourne, L. E., Jr. Knowing and using concepts. Psychological Review, $1970,77,546-556$.

Bourne, L. E., Jr., \& Guy, D. E. Learning conceptual rules: I. Some interrule transfer effects. Journal of Experimental Psychology, 1968a, 76, 423-429.

Bourne, L. E., Jr., \& Guy, D. E. Learning conceptual rules: II. The role of positive and negative instances. Journal of Experimental Psychology, 1968b, 77, 488-494.

Bower, A. C., \& King, W. L. The effect of number of irrelevant stimulus dimensions, verbalizations, and sex on learning. Psychonomic Science, 1967, 8, 453-454.

Conant, M B \& Trabasso, T. Conjunctive and disjunctive concept information under equal-information conditions. Journal of Experimental Psychology, 1964, 57, 250-255.

Di Vesta, F. J., \& Walls, R. T. Rule and attribute identification in children's attainment of conjunctive and disjunctive in children's attinment of Experimental Psychology, 1969, 80, 498-504.

Haygood, R. C., \& Bourne, L. E., Jr. Attribute- and rule-learning aspects of conceptual behavior. Psychological Review, 1965, 72, 175-195.

Haygood, R. C., \& Stevenson, M. Effects of number of irrelevant dimensions in nonconjunctive concept learning. Journal of Experimental Psychology, 1967, 74, 302-304. 
Kepros, P. G., \& Bourne, L. E., Jr. Identification of biconditional concepts: Effects of number of relevant and irrelevant dimensions. Canadian Journal of Psychology, 1966 30, 198-207.

Laughlin, P. R. Focusing strategy for eight concept rules. Journal of Experimental Psychology, 1968, 77, 661-669.

Laughlin, P. R., \& Jordan, R. M. Selection strategies in conjunctive, disjunctive, and biconditional concept attainment. Journal of Experimental Psychology, 1967, 75, 188-193.
Taplin, J. E., \& Jeeves, M. A. Strategies in concept learning. Program on Concept Learning Report No. 19, Institute for the Study of Intellectual Behavior, University of Colorado, 1972, 1-54.

(Received for publication February 16, 1974.)

\title{
Differential conditioning and contrast effects in humans*
}

\author{
RICHARD S. CALEF $\dagger$, RUTH ANN CALEF, GRANT BUTTERMORE, and SUSAN J. THOMAS \\ West Virginia Wesleyan College, Buckhannon, West Virginia 26201
}

\begin{abstract}
The present study investigated discrimination $\mathrm{S}+$ contrast effects as a function of the magnitude of reward in $\mathrm{S}$ - while attempting to alleviate ceiling effect and decision time problems present in previous animal and human simultaneous contrast studies. In the study, three groups of human Ss recrived differential reward conditioning for accurately tracing numbered-nonnumbered star patterns, whereas a control group received only large reward in both discriminanda. The results replicated the findings of a previous animal study which also attempted to control for ceiling and decision time variables in that a positive S+ contrast effect was found. Also, the positive S+ contrast effect increased as the magnitude of reward in $\mathrm{S}$ - decreased. The results seem to support a perceptual analysis of contrast effects.
\end{abstract}

Previous studies (e.g., Bower, 1961; Ludvigson \& Gay, 1966) have shown that performance of rats to the negative discriminandum $\left(\mathrm{S}_{-}\right)$in differential conditioning is influenced by the reinforcement contingencies associated with the positive discriminand um $(\mathrm{S}+)$. Generally, these studies demonstrate that the $\mathrm{S}$ - performance of differentially

*This paper is sponsored by James H. McHose, who takes full editorial responsibility for it.

†Requests for reprints should be sent to Richard S. Calef, Department of Psychology, West Virginia Wesleyan College, Buckhannon, West Virginia 26201. reinforced (discrimination) groups is depressed relative to that of nondiscrimination control groups receiving small reinforcement in "S+" and "S-" (negative Scontrast). Previous studies have also shown a significant depression (Henderson, 1966; MacKinnon, 1967) of S+ speeds for discrimination Ss relative to that of nondiscrimination control Ss receiving large reinforcement in "S+" and "S-" (negative S+ contrast).

Little information has been available concerning how human Ss respond to stimuli associated with contrasting magnitude of reward. Recently, however, Calef et al 